ARTICLE

https://doi.org/10.1038/s41467-019-12112-w

\title{
EMS1 and BRI1 control separate biological processes via extracellular domain diversity and intracellular domain conservation
}

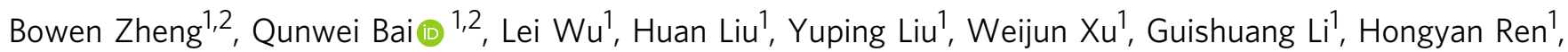
Xiaoping She ${ }^{1} \&$ Guang Wu (1) ${ }^{1}$

In flowering plants, EMS1 (Excess Microsporocytes 1) perceives TPD1 (Tapetum Determinant 1) to specify tapeta, the last somatic cell layer nurturing pollen development. However, the signaling components downstream of EMS1 are relatively unknown. Here, we use a molecular complementation approach to investigate the downstream components in EMS1 signaling. We show that the EMS1 intracellular domain is functionally interchangeable with that of the brassinosteroid receptor BRI1 (Brassinosteroid Insensitive 1). Furthermore, expressing EMS1 together with TPD1 in the BR11 expression domain could partially rescue bri1 phenotypes, and led to the dephosphorylation of BES1, a hallmark of active BRI1 signaling. Conversely, expressing BRI1 in the EMS1 expression domain could partially rescue ems1 phenotypes. We further show that PpEMS1 and PpTPD1 from the early land plant Physcomitrella patens could completely rescue ems1 and tpd1 phenotypes, respectively. We propose that EMS1 and BRI1 have evolved distinct extracellular domains to control different biological processes but can act via a common intracellular signaling pathway.

\footnotetext{
${ }^{1}$ College of Life Sciences, Shaanxi Normal University, 710119 Xi'an, Shaanxi Province, China. ${ }^{2}$ These authors contributed equally: Bowen Zheng, Qunwei Bai. Correspondence and requests for materials should be addressed to X.S. (email: shexiaoping@snnu.edu.cn) or to G.W. (email: gwu3@snnu.edu.cn)
} 
$\mathrm{n}$ angiosperms, the development of male reproductive organs is precisely controlled to achieve successful fertilization and reproduction ${ }^{1-4}$. The tapetum, which is required for pollen production, provides pollen nutritive support, remodels the callose coat surrounding microsporocytes and tetrads, and synthesizes most components of the pollen wall ${ }^{2,5-9}$. Anthers in ems 1 (Excess Microsporocytes 1), also known as exs (Extra Sporogenous Cells), and tpd1 (Tapetum Determinant 1) mutants have no tapetal cells; instead, they produce excess microsporocytes $6,10,11$. TPD1 is secreted from microsporocyte precursors and then activates EMS1, which is localized at the plasma membrane of tapetal precursor cells/tapetal cells 7,12 . The EMS1-TPD1 signaling pathway initially promotes periclinal division of parietal cells to form tapetal precursor cells, and later determines and maintains the fate of functional tapetal cells ${ }^{7,12}$. The SERK1/2 (Somatic Embryogenesis Receptor-Like Kinase 1,2) LRR-RLKs (Leucine Rich Repeat Receptor-Like Kinases), act as potential co-receptors of EMS 13 .

Given the significance of EMS1 in male fertility, identifying its downstream signaling components is critical. However, since no homozygous seeds can be obtained from ems1 null mutants, isolation of its downstream components via genetic screens is complicated $^{10,11}$. Furthermore, as EMS1 expression is tapetumspecific and ems 1 mutants have no tapeta, molecular isolation of downstream components is difficult. Moreover, yeast two-hybrid screen can only isolate direct interactors and affinity purification may generate non-specificity ${ }^{14-16}$. Thus, it was until last year that a putative substrate of EMS1, a family of $\beta$-carbonic anhydrases $(\beta \mathrm{CAs})$, was identified ${ }^{17}$. However, the way $\beta$ CAs transmit the EMS1 signal to downstream targets is completely unknown ${ }^{17}$. Therefore, identifying additional EMS1-TPD1 signaling components is a necessary but challenging task.

EMS1 belongs to the LRR-X (Leucine Rich Repeats-X) subfamily of receptor-like kinases (RLKs), the largest family of cell surface receptors in land plants ${ }^{18}$. The LRR-RLK-X subfamily also includes BRI1 (Brassinosteroid Insensitive 1) and PSKR1 (Phytosulfokine Receptor 1). Thus, EMS1, BRI1 and PSKR1 have high sequence similarity ${ }^{18-21}$. However, these receptors have distinct biological functions ${ }^{10,11,20,21}$. Current knowledge suggests that RLKs use their versatile extracellular domains (ECDs) to perceive a variety of ligands, which activate conserved intracellular kinase domains (ICDs) to regulate different downstream targets and control distinct biological processes ${ }^{18,19}$. Thus, with greater divergence of the ECDs relative to the $\operatorname{ICDs}^{18,22}$, it is possible that the ECDs could bind different ligands, while their ICDs still target the same downstream components. This together with differential gene expression controls diverse biological functions ${ }^{18}$. It has been demonstrated that the ICD of BRI1 can be activated in chimeric receptors with the ECDs of distinct RLKs that perceive non-BR ligands or that are coreceptor kinases 22,23 . This finding indicates that the same ECD can activate different ICDs while different ECDs can also activate the same ICD, providing a technical framework to functionally study ECDs and ICDs of a variety of RLKs using chimeric receptors.

Among all the RLKs, BRI1 is one of the best studied receptors. Brassinosteroids (BRs) bind directly to the ECD of BRI1 to activate its ICD, thus conferring a BR-specific function ${ }^{22,24-26}$. After binding BRs, BRI1 interacts with BAK1 (BRI1 Associate Kinase 1) and SBI1 (Suppressor of bri1 1) ${ }^{27-29}$. This activates BRI1 to phosphorylate BSK1 (BR-Signaling Kinase 1) and BRI1specific substrate (BKI1, BRI1-Kinase inhibitor 1) ${ }^{30,31}$, which successively releases BKI1 and inactivates the negative regulator, BIN2 (Brassinosteroid Insensitive 2) ${ }^{32,33}$. This then activates BSU1 (bri1 Suppressor 1) and BES1/BZR1 (bri1 EMS-Suppressor 1)/(Brassinozole Resistant 1) transcription factors to regulate plant growth and development ${ }^{34-36}$. Finally, activated BES1/
BZR1 regulates the expression of numerous BR responsive genes $^{37,38}$.

ems1 null mutants appear almost normal, but lack pollen, while bri1 null mutants display extreme dwarfism with almost normal pollen ${ }^{10,11,39}$, implying their non-overlapping biological functions $10,11,21,40$. In this work, we show that the BRI1 and EMS1 intracellular domains are functionally exchangeable. We find that expression of BRI1 in the EMS1 expression domain and co-expression of EMS1 and TPD1 in the BRI1 expression domain can partially complement ems 1 and bri1 mutants, respectively, suggesting that they can activate the same downstream components. We show that EMS1 and BRI1 originated in early land plants and flowering plants, respectively, and suggest a route for functional divergence of RLKs.

\section{Results}

The intracellular domains of EMS1 and BRI1 are interchangeable. To identify potential downstream signaling molecules of EMS1 in the tapeta, we used a molecular complementation approach, considering sequence homology and evolutionary conservation to design domain swaps (Supplementary Fig. 1). The RLK family arose from a common ancestor and has since expanded by gene duplication and divergence ${ }^{18,19}$. Thus, RLKs might share similar downstream components even though they control very different biological functions. Therefore, the successful substitution of an ICD of one RLK with that of another RLK ICD could imply common targets. To test this notion, we fused the BRI1 ECD with the ICD of EMS1 and other RLKs to generate chimeric receptors that can perceive BRs to activate the intracellular function of each respective RLK (Fig. 1a). These engineered receptors were introduced into the plants under the control of BRI1 promoter. We found that only the expression of pro ${ }^{B R I 1}:: E C D^{B R I 1}-I C D^{E M S 1}$ (BRI1-EMS1), but not pro ${ }^{B R I 1::}$ $E C D^{B R I 1}-I D^{P S K R 1}$ (BRI1-PSKR1), nor any other pro $^{B R I 1}:: E C D$ $B R I 1_{-I C D}{ }^{R L K s}$ (BRI1-RLKs), rescued bri1 mutant phenotypes (Fig. 1a-c and Supplementary Figs. 1, 2a, b and 3a, b), consistent with the hypothesis that both EMS1 and BRI1 ICDs can activate common targets.

To test whether the BRI1-EMS1 chimera indeed activate BRdependent targets, we exogenously applied brassinolide (BL) and brassinazole (BRZ), a BR biosynthesis inhibitor that blocks the production of $\mathrm{BRs}^{41}$, to the transgenic seedlings. This showed that they had similar sensitivity to BR as that of wild type (WT) or transgenics expressing BRI1 (Supplementary Fig. 3c-f). These results indicate that the chimeric BRI1-EMS1 is able to functionally substitute for BR receptors, probably acting through similar downstream molecules as BRI1. Accumulation of dephosphorylated BES1/BZR1 upon BR treatment is indicative of active BR signaling 35,36 , and we detected higher accumulation of dephosphorylated BES1 in BRI1-EMS1/bri1 transgenics compared to bri1 mutants. To test whether the accumulation of dephosphorylated BES1 is due to activation of the chimeric BRI1EMS1 receptor by BRs, we treated seedlings with $\mathrm{BL}$ and found further accumulation of dephosphorylated BES1 in the BRI1EMS1/bri1 transgenics but not in bri1 mutants (Fig. 1d). Additionally, the expression of other BR regulated genes was also restored compared to WT and bril mutants (Supplementary Fig. 3g-i). Altogether, our results indicate that chimeric BRI1EMS1 can functionally substitute for BRI1 when expressed in the BRI1 expression domain.

As EMS1-TPD1 downstream signaling events are almost unknown in the tapeta, we asked whether the BRI1 ICD could replace that of EMS1 in the tapeta. BRI1 and PSKR1 ICDs were fused with the EMS1 ECD and the chimeric receptors were expressed in ems 1 null mutant backgrounds under the control of 
a

\begin{tabular}{lllll} 
BRI1 & ECD & ID & TM & ICD \\
BRI1-EMS1 & ECD & ID & TM & ICD \\
BRI1-PSKR1 & ECD & ID & ICD \\
BRI1-GSO1 & ECD & ID & IM \\
BRI1-CLV1 & ECD & ID & TM & ICD \\
BRI1-BAM1 & ECD & ID & TM & ICD \\
BRI1-EFR & ECD & ID & TM & ICD \\
BRI1-BAK1 & ECD & ID & TM & ICD \\
\hline
\end{tabular}

d

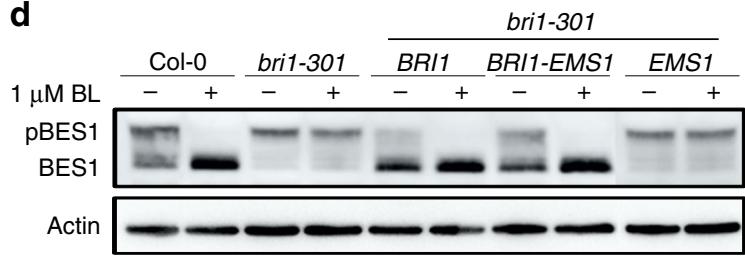

$\mathbf{e}_{\text {EMS1 }}$

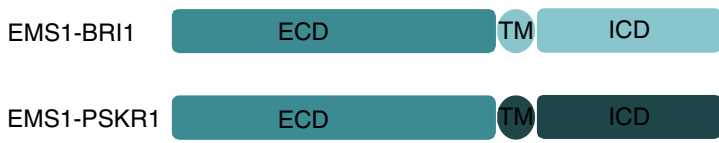

\section{g}

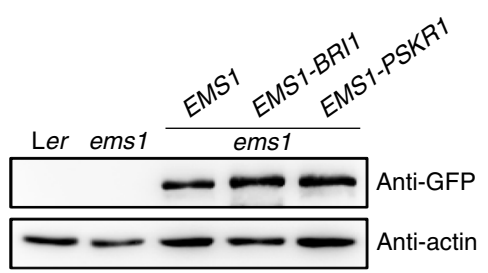

b

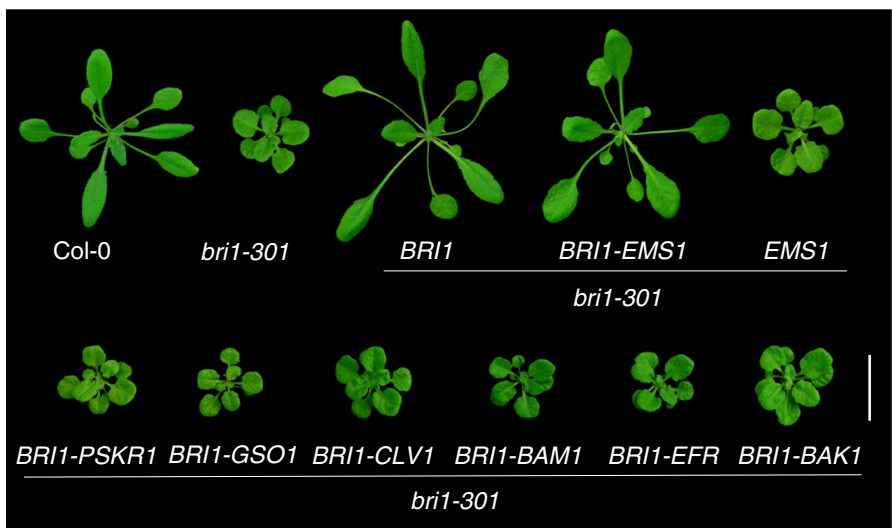

C

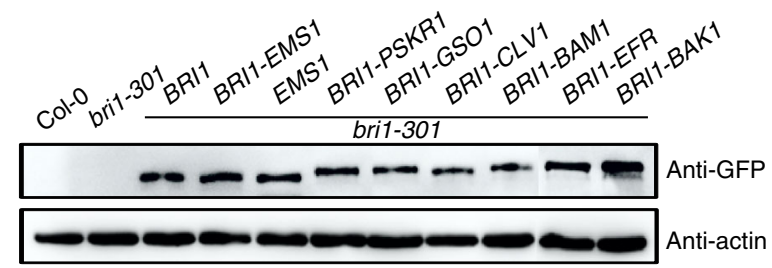

$\mathbf{f}$
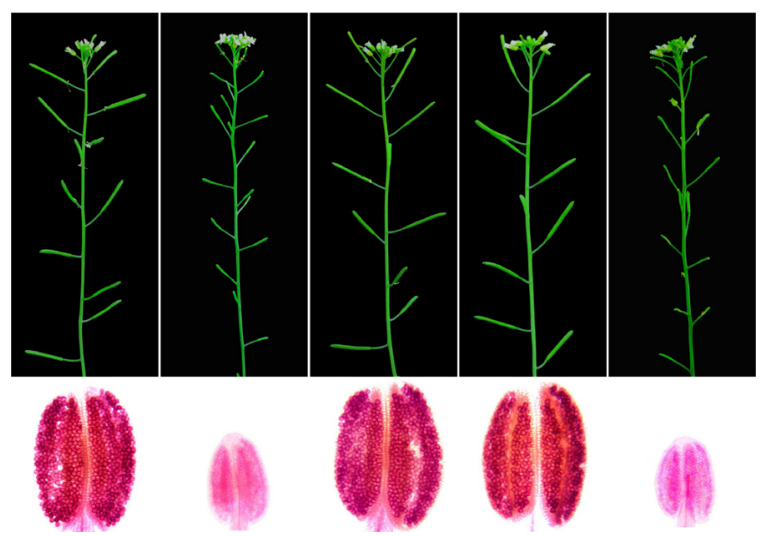

Ler

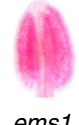

EMS1

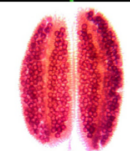

EMS1-BR

Fig. 1 The intracellular domains of EMS1 and BRI1 are interchangeable. a Schematic diagram of the extracellular domain of BRI1 fused with the transmembrane domains and intracellular kinase domains of several LRR-RLKs that were labeled in different colors, respectively. $\mathbf{b}$ Phenotypes of 4-weekold transgenic lines expressing BRI1, BRI1-EMS1, EMS1, BRI1-PSKR1, BRI1-GSO1, BRI1-CLV1, BRI1-BAM1, BRI1-EFR and BRI1-BAK1 under the BRI1 promoter in bri1301 background. Scale bar, $2.0 \mathrm{~cm}$. c Protein expression levels of the transgenes with GFP tag in the rosette leaves of the corresponding plants shown in $\mathbf{b}$ were detected with anti-GFP antibody. Actin served as the loading control. d Phosphorylated BES1 (pBES1) and dephosphorylated BES1 were detected with BES1 antibodies in the extracts of 10-day-old seedlings of the indicated genotypes. Where indicated, the plants were treated with $1 \mu \mathrm{M} B \mathrm{BL}$ for $1 \mathrm{~h}$ before preparation of the extracts. Actin served as the loading control. e Schematic diagram of chimeric receptor kinases EMS1-BRI1, EMS1-PSKR1. The EMS1, BRI1 and PSKR1 protein structures were labeled in blue, light blue and dark blue, respectively. $\mathbf{f}$ Phenotypes of 6-week-old transgenic lines expressing EMS1, EMS1-BRI1, EMS1-PSKR1 under the EMS1 promoter in ems1. Primary inflorescences (top) and Alexander staining of pollen grains in mature anthers (bottom) showing the fertility phenotypes of the transgenic plants. $\mathbf{g}$ Protein expression levels of the transgenes with GFP tag in the inflorescences of the corresponding plants shown in $\mathbf{f}$ were detected with anti-GFP antibody. Actin served as the loading control

EMS1 promoter. The expression of $\operatorname{pro}^{E M S 1}:: E C D^{E M S 1}-I C D^{B R I 1}$ (EMS1-BRI1) but not pro ${ }^{E M S 1:: E C D^{E M S 1}-I C D^{P S K R 1}}$ (EMS1PSKR1) completely rescued the ems 1 mutant phenotypes (Fig. 1e-g and Supplementary Fig. 2c, d). This suggests that the signaling events that occur downstream of EMS1 may be activated by the BRI1 ICD in tapeta. Taken together, our results suggest that the ICDs of EMS1 and BRI1 are interchangeable.

EMS1-TPD1 and BRI1-BRs receptor-ligand pairs can crosscomplement. In plants, EMS1-TPD1 and BRI1-BRs control completely different biological processes, yet the BRI1 and EMS1 ICDs are functionally interchangeable, implying that they might share common downstream signaling events. To test this hypothesis further, we individually expressed EMS1 and TPD1 under the control of BRI1 promoter in Arabidopsis. Both failed to rescue bri1 mutant phenotypes. However, co-expression of proBRI1::EMS1 and pro ${ }^{B R I 1:: T P D 1}$ (EMS1 \& TPD1) together partially rescued the dwarf stature phenotype of the bril mutant (Fig. 2a-c, e, $\mathrm{f}$ and Supplementary Figs. $4 \mathrm{a}-\mathrm{d}$ and $5 \mathrm{a})$. Yet, pro $^{\text {BRII }:: E M S 1}$ and pro $^{B R I 1:: T P D 1 / b r i 1}$ co-expressing plants still had shorter 
a

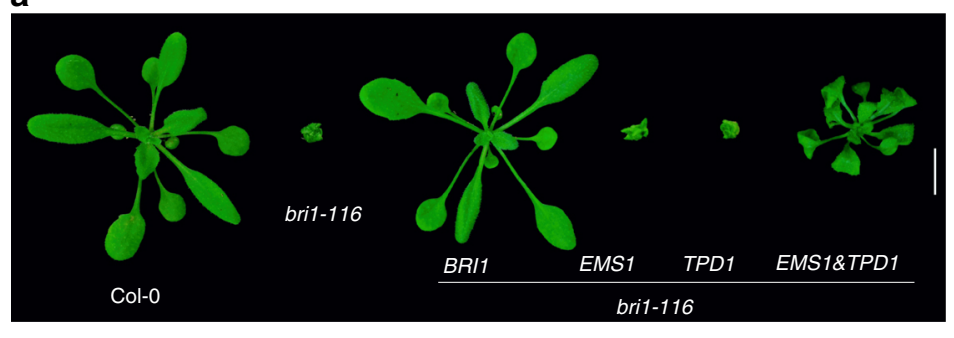

b

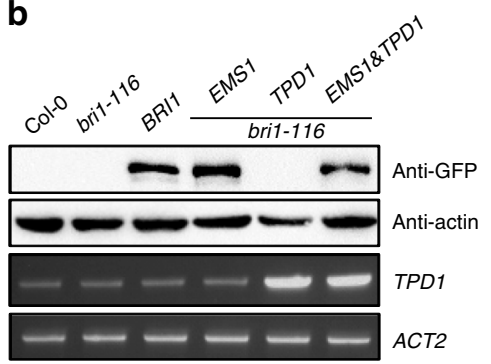

C

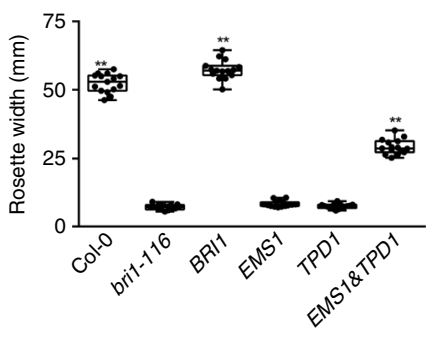

d

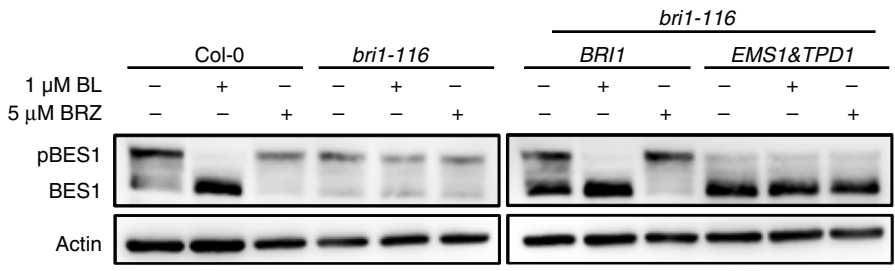

e
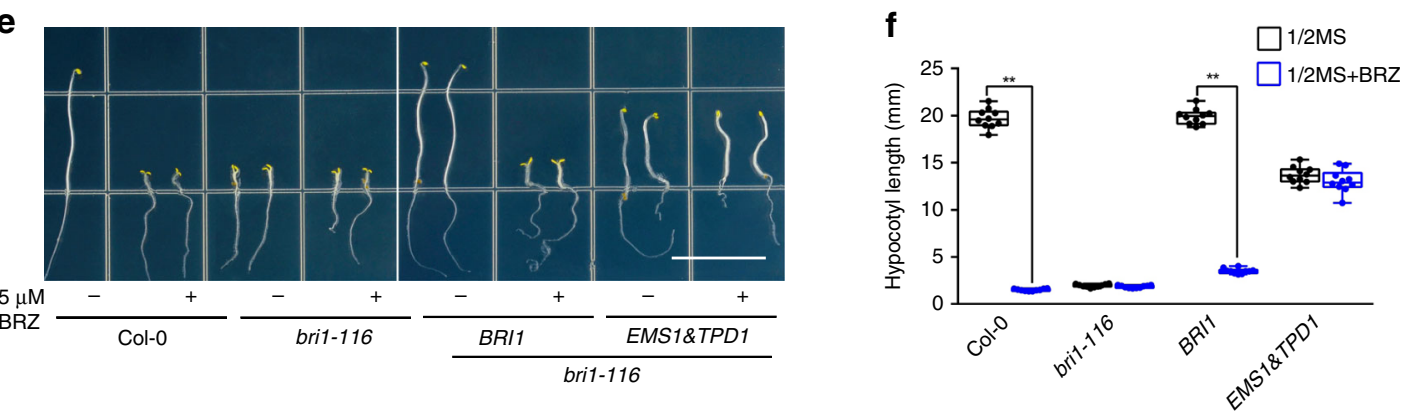

g

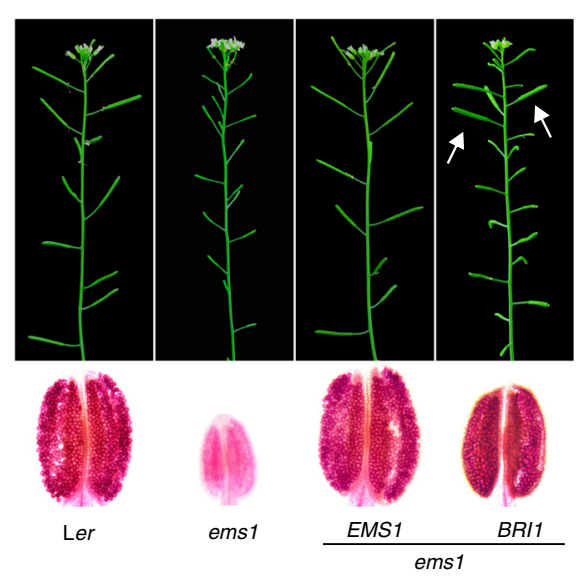

h

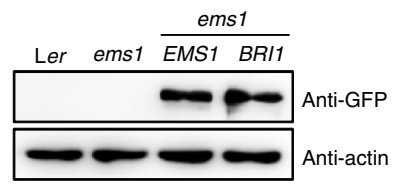

hypocotyls, smaller rosette diameters, curled and epinastic leaves, delayed senescence, reduced male fertility, and smaller siliques with fewer seeds compared to WT plants (Fig. 2a-f and Supplementary Figs. 4a-d and 5). Furthermore, pro ${ }^{B R I 1}:: E M S 1$ and

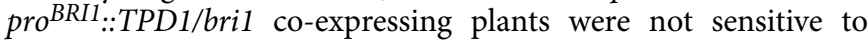
BRs, as was shown by treatment with BL and BRZ (Fig. 2d-f), confirming that the partial rescue was BR independent. To test if this functional recovery is correlated with activation of signaling components downstream of BRI1, we examined the accumulation and phosphorylation status of BES1 in the co-expression plants. Significantly, we detected accumulation of dephosphorylated

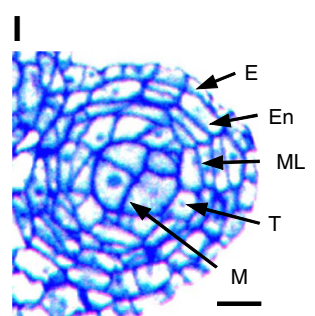

Ler

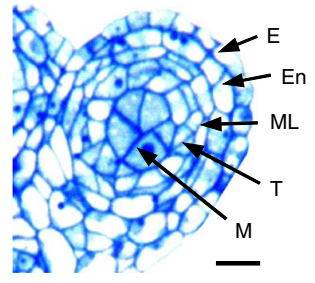

EMS1/ems1

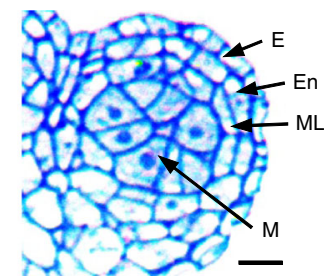

ems 1

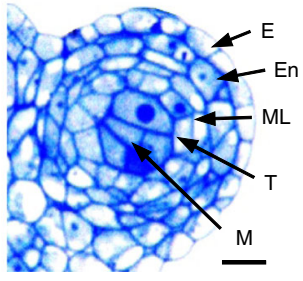

BRI1/ems1
BES1 in the co-expression lines compared to bri1 mutants and this was independent of BRs (Fig. 2d and Supplementary Fig. 4a-d). We noticed a correlation between the accumulation of dephosphorylated BES1 and plant phenotypes, although it was non-linear (Fig. 2d and Supplementary Fig. 4a-d), possibly suggesting regulatory feedback. Nevertheless, our results indicate that BES1 can be activated independently of BRs by ectopic expression of EMS1-TPD1.

To further assess the similarity between ectopic EMS1-TPD1 signaling and native $\mathrm{BR}$ signaling, we used quantitative real-time PCR to analyse the expression of BR biosynthetic genes $C P D$ and 
Fig. 2 EMS1-TPD1 and BRI1-BRs receptor-ligand pairs can cross-complement. a The phenotypic recovery of bri1-116 null allele by expressing BRI1, TPD1, EMS1 independently and EMS1 together with TPD1 under the control of BRI1 promoter. Scale bar, $1 \mathrm{~cm}$. $\mathbf{b}$ Analyses of the expression levels of the transgenes in the rosette leaves of the corresponding plants shown in a Protein expression levels were detected with anti-GFP antibody. Actin served as the loading control. TPD1 expression levels were detected by semi-quantitative RT-PCR. ACT2 served as an internal control. c Quantification of the transgenic lines with the diameter of the rosette leaves in the whole plants grown for 4 weeks, $n=15$ plants, ${ }^{\star} P<0.05,{ }^{\star} *<0.0001$ as one-way ANOVA with a Tukey's test. $\mathbf{d}$ Dephosphorylation of BES1 by both BRI1-BRs and EMS1-TPD1 signaling pathways. Expression of BRI1, or EMS1 together with TPD1 under the control of BR/1 promoter rescued the dephosphorylation of BES1 in BR-dependent and BR-independent manner, respectively. BL brassinolide, BRZ brassinozole.

Phosphorylated BES1 (pBES1) and dephosphorylated BES1 were detected with BES1 antibodies in the extracts of 10-day-old seedlings of the indicated genotypes. e, $\mathbf{f}$ Co-expression of EMS1 \& TPD1 in bri1-116 partially rescued the hypocotyl elongation of bri1-116 mutant but did not restore the sensitivity to BRZ. 5-day-old dark-grown seedlings in $1 / 2$ MS medium treated with or without $5 \mu$ M BRZ. Scale bar, $1.5 \mathrm{~cm}$. $n=10$ seedlings. ${ }^{\star \star} P<0.0001$ (two-way ANOVA with Sidak's test). $\mathbf{g}$ Anthers with or without pollen grains in the transgenic lines expressing EMS1 or BR/1 under the control of EMS1 promoter in ems1 background. Arrows, fertile siliques. h Protein expression levels of the transgenes with GFP tag were detected in the inflorescences of the corresponding plants shown in $\mathbf{g}$ with anti-GFP antibody. Actin served as the loading control. $\mathbf{i}$ Semi-thin sections of stage- 5 anthers showing the normal anther cell differentiation in Ler, proEMS1::EMS1 in ems1 or proEMS1::BRI1 in ems1 as well as the abnormal anther cell differentiation in ems1 (lack of tapetal cells). E epidermis, En endothecium, ML the middle layer, T tapetal cells, and M microsporocytes. Scale bars, $10 \mu \mathrm{m}$

DWF4 or BR catabolic gene BAS1 in the transgenic plants of pro $^{B R I 1 .: E M S 1}$ and pro $^{B R I 1:: T P D 1 / \text { bri1 co-expression lines. } C P D}$ and DWF4 were dramatically downregulated while BAS1 was upregulated to resemble that of WT (Supplementary Fig. 5c-e). These data indicate that ectopic EMS1-TPD1 signaling can trigger a similar expression response to native BRI1-BR signaling. These results also suggest that BRI1-BR signaling is largely replaceable by EMS1-TPD1 when expressed in the BRI1 expression domain and that similar downstream signaling events are activated.

If EMS1-TPD1 and BRI1-BRs do share key downstream events, then their replacement should be bidirectional. BRs are small hydrophobic chemicals, which may be difficult to restrict in between tapeta cell layers and microsporocytes in the manner of $20 \mathrm{kDa}$ protein TPD $1^{12,42}$. However, pollen is a rich source of endogenous BRs, meaning that it likely has high level of free BRs in the anthers ${ }^{43}$. We reasoned if BRs were enriched during pollen development, and if BRI1 was in the tapeta, it could access BRs to initiate BR signaling in tapeta, thus suppressing the ems 1 mutant phenotypes if the EMS1-TPD1 function can be replaced by BRI1BR function. To test this hypothesis, we expressed the BRI1 receptor in tapeta by introducing the $B R I 1$ gene under the control of EMS1 promoter into the ems 1 mutant plants. Indeed, the expression of pro ${ }^{E M S 1}:$ :BRI1 largely suppressed ems1 mutant phenotypes (Fig. 2g-i and Supplementary Fig. 4e-f). Furthermore, we observed that the transgenic plants had viable pollen and normal seeds, although they had fewer and shorter siliques (Fig. 2g-i and Supplementary Fig. 4e, f). Significantly, pro ${ }^{E M S 1}:$ : BRI1/ems1 plants had normal tapetal cells while ems 1 had none (Fig. 2i). Altogether, this suggests that EMS1-TPD1 is partially replaceable by BRI1-BRs in tapetum development.

Dominant BR signaling mutants can modulate EMS1 signaling. To further investigate the downstream components in EMS1-TPD1 signaling, we took advantage of two available dominant mutants $b z r 1-1 D$ and bes1-D that suppress nearly all phenotypic defects of the bri1 mutants. bzr1-1D and bes1-D are point mutation mutants, where both active (dephosphorylated) and inactive (phosphorylated) BES1/BZR1 accumulate, causing BR signaling to be amplified with or without $\mathrm{BRs}^{35,36}$. We reasoned that if EMS1-TPD1 functions through BES1/BZR1, then the tapeta may develop without EMS1-TPD1 in these mutants. Thus, we crossed bzr1-1D and bes1-D mutants with ems1 and tpd1 single mutants and serk1serk2 double mutants. We found that all double or triple mutants, ems1bzr1$1 D$, ems1bes1-D, tpd1bzr1-1D, tpd1bes1-D, serk1serk2bzr1-1D or serk1serk2bes1-D, exhibited normal tapeta, pollen and seeds (Fig. 3a, b and Supplementary Fig. 6), consistent with our hypothesis. However, the native BZR1 and BES1 are ubiquitously expressed in plants and there is a possibility that these dominant effects are indirect. We thus expressed the bzr1-1D and bes1-D point mutant variants under the EMS1 promoter in the ems1 mutants, and found that they exhibited similar phenotypes as the dominant genetic mutants (Supplementary Fig. 6g, h), confirming that BZR1 and BES1 can indeed promote tapetum development when expressed in the EMS1 expression domain.

BIN2 is a repressor of BRI1-BR signaling, and bin2-1D mutant phenotypes resemble those of bri1 mutants ${ }^{32}$. To determine if BIN2 can also suppress the EMS1-TPD1 signaling pathway, we co-expressed TPD1 and EMS1 under the BRI1 promoter in the bin2-1D mutant background. We found that the EMS1 with TPD1 expression could not overcome the inhibitory effects of the bin2-1D mutants (Fig. 3c, d), suggesting that BIN2 can also act downstream of EMS1-TPD1.

EMS1 and BRI1 signaling complementarily control the Arabidopsis life cycle. To further understand the role of EMS1-TPD1 and BRI1-BRs in the control of plant development, bri1-116 null mutants were crossed to ems 1 mutants to generate bri1-116ems 1 double mutants. We found that the double mutants had an additive effect of both single mutant phenotypes, resulting in dwarf plants lacking tapeta and mature pollen (Supplementary Fig. 7a). This suggests that EMS1-TPD1 and BRI1-BRs independently control separate developmental processes in Arabidopsis. To further test their independence, we expressed pro ${ }^{B R I 1}$ :: EMS1 in ems1 mutants and found that it did not suppress ems 1 mutant phenotypes (Supplementary Fig. 7b, c). Consistently, we observed stronger expression of GFP in the tapetal cell layer of transgenic plants expressing pro ${ }^{E M S 1}:: E M S 1:: G F P$ than in transgenic plants expressing pro ${ }^{B R I 1}:: E M S 1:: G F P$ (Supplementary Fig. 7d), suggesting little or no BRI1 accumulation in the tapeta.

Interestingly, null bes1/bzrl family mutants are dwarf and lack pollen resembling ems 1 bril double mutants ${ }^{44}$. This is consistent with a role for the native BES1/BZR1 family controlling tapetal cell fate downstream of EMS1-TPD1.

EMS1-TPD1 are conserved across land plants. To investigate the conservation of EMS1-TPD1 signaling in terrestrial plants, we studied Physcomitrella patens, one of the earliest land plant species lacking true tapeta ${ }^{45}$. We found that EMS1 and TPD1 are present in completely sequenced genomes of all land plants but not in alga (some examples were shown in Supplementary Fig. 8) ${ }^{45-51}$. P. patens $(P p)$ had EMS1 (PpEMS1) and TPD1 (PpTPD1) but not BRI1 or BRI1-likes (BRLs) $21,26,45,51-54$. There were six PpEMS1 and one PpTPD1 in P. patens (Supplementary Fig. 8). To determine whether they have functional ICDs, we 
a
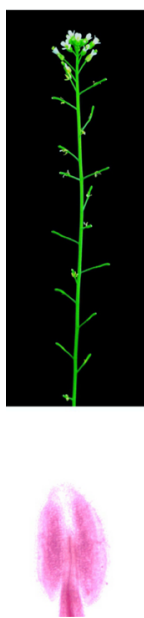

ems 1
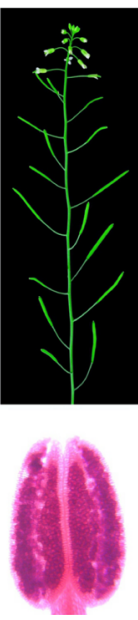

bzr1-1D ems1 bes $1-D$ ems 1
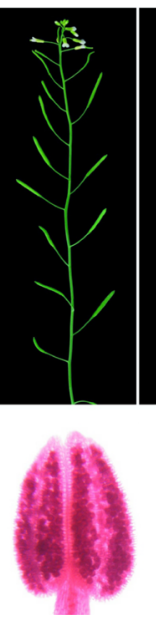
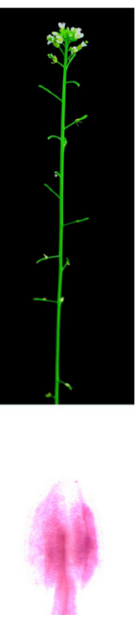

tpd1
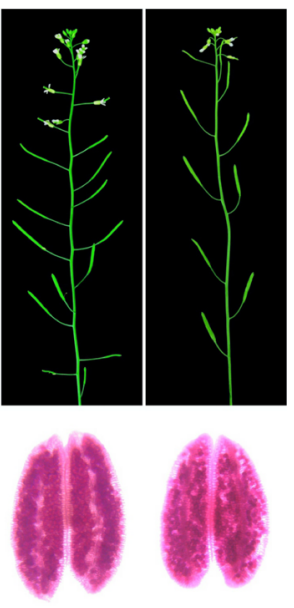

bzr1-1D tpd1 bes1-D tpd1 b

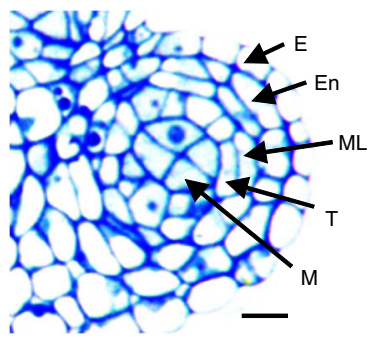

bzr1-1D ems 1

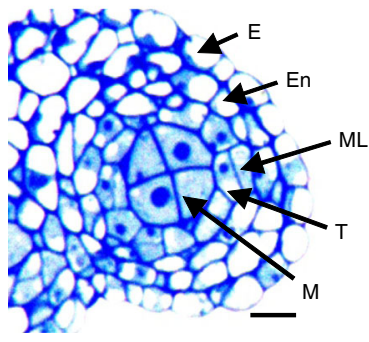

bzr1-1D tpd1

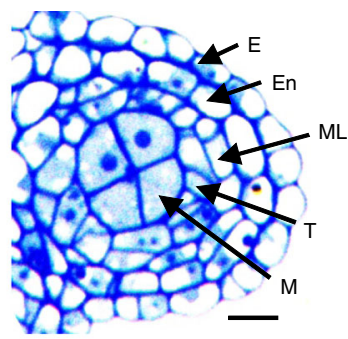

bes $1-D$ ems 1

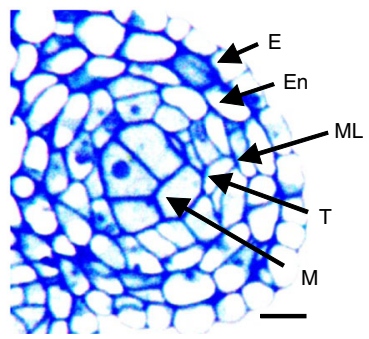

bes $1-D$ tpd1

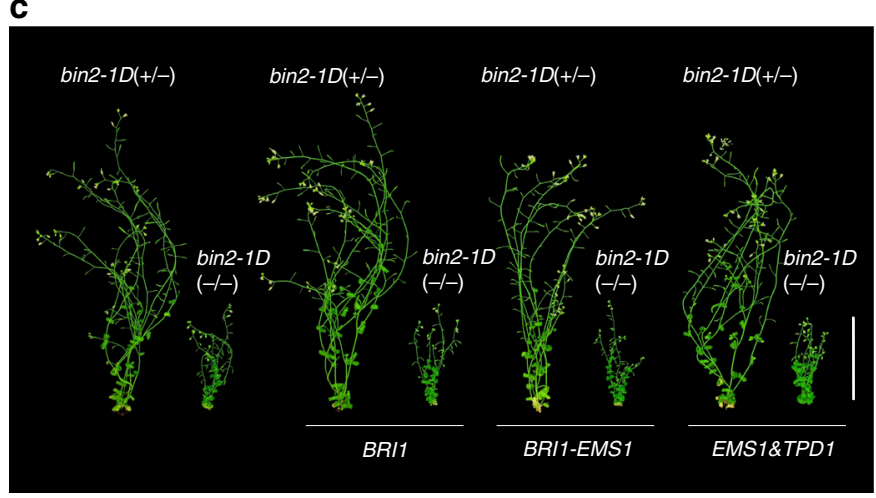

d

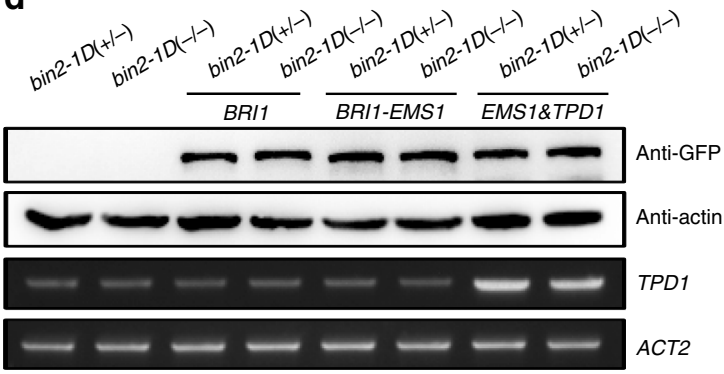

Fig. 3 Activated transcription factors of BRI1-BRs signaling can rescue ems1 and tpd1 mutants. a bes1-D and bzr1-1D rescued the male sterile phenotypes of ems 1 and tpd1 mutants. Primary inflorescences (top) and Alexander staining of pollen grains in mature anthers (bottom) showing the fertility phenotypes of bzr1-1Dems1, bes1-Dems1, bzr1-1Dtpd1 and bes1-Dtpd1 double mutants. b Semi-thin sections of stage-5 anther lobes showing four somatic cell layers, including epidermis (E), endothecium (En), the middle layer $(\mathrm{ML})$ and tapetum $(\mathrm{T})$, as well as reproductive microsporocytes $(\mathrm{M})$ in the center of bzr1-1Dems1, bes1Dems1, bzr1-1Dtpd1 and bes1-Dtpd1 double mutants, respectively. Scale bars, $10 \mu \mathrm{m}$. c BIN2 inhibited EMS1-TPD1 signaling. Phenotypes of 7-week-old transgenic plants expressing BRI1, BRI1-EMS1, EMS1 and TPD1 under the control of BRI1 promoter in bin2-1D (+/ -) and bin2-1D (-/-) plants. Scale bar, 5 $\mathrm{cm}$. d Analyses of the expression levels of the transgenes in the rosette leaves of the corresponding plants shown in c. Proteins with GFP tag were detected with anti-GFP antibody. Actin served as a loading control. TPD1 expression was detected by semi-quantitative RT-PCR. ACT2 served as an internal control

fused the BRI1 ECD with the six PpEMS1 ICDs. All of these chimeric constructs suppressed the phenotypes of bri1 mutants when expressed under the BRI1 promoter (Supplementary Fig. 9). Furthermore, the expression of PpEMS1-1 and PpEMS1-2 under the control of the EMS1 promoter completely rescued ems 1 phenotypes. Meanwhile, PpTPD1 expression under the TPD1 prompter in tpd1 mutant completely recued tpd1 phenotypes (Fig. 4a, b). Importantly, co-expression of either PpEMS1-1 or PpEMS1-2 with PPTPD1 under the BRI1 promoter in bri1 mutants partially suppressed the bri1 dwarf phenotype and caused accumulation of dephosphorylated BES1 (Fig. 4c-e). As expected, these transgenic seedlings were not sensitive to BRZ treatment, showing no retardation in hypocotyl growth (Fig. 4f-g).

Altogether, these findings suggest that EMS1 and TPD1 have been able to form a functional pair for at least 400 million years ${ }^{46,55}$. Since BRI1 and BRLs were not found in P. patens or other early land plants, such as liverworts ${ }^{46}$, this implies that BR signaling emerged after EMS1-TPD1 signaling. Phylogenetic analysis revealed that BR receptors shared common ancestors with EMS1 (ref. ${ }^{56}$ ).
Significantly, BRI1 was not found in any non-flowering plants with completely sequenced genomes, including liverworts, mosses, lycophytes, ferns and gymnosperms ${ }^{45-50,56,57}$, implying that BRI1 was rapidly neofunctionalized in angiosperms, and likely attributing to the adaptive advantage of flowering plants. Altogether, we propose that expansion of BRI1/EMS1 signaling may have accompanied land plant evolution. EMS1 may be important for all land plants whereas BRI1 appears only essential in flowering plants (Fig. 4h).

\section{Discussion}

Cell-to-cell communication is essential for cell differentiation and development, which is largely controlled by cell surface receptors that perceive and transmit various signals from the outside into the inside of the cell to control a wide range of physiological and developmental processes ${ }^{4}$. RLKs are the largest family of cell surface receptors in land plants. In Arabidopsis alone, there are more than 600 RLKs, accounting for $2.5 \%$ of all coding proteins, $60 \%$ of all kinases and nearly all 
a

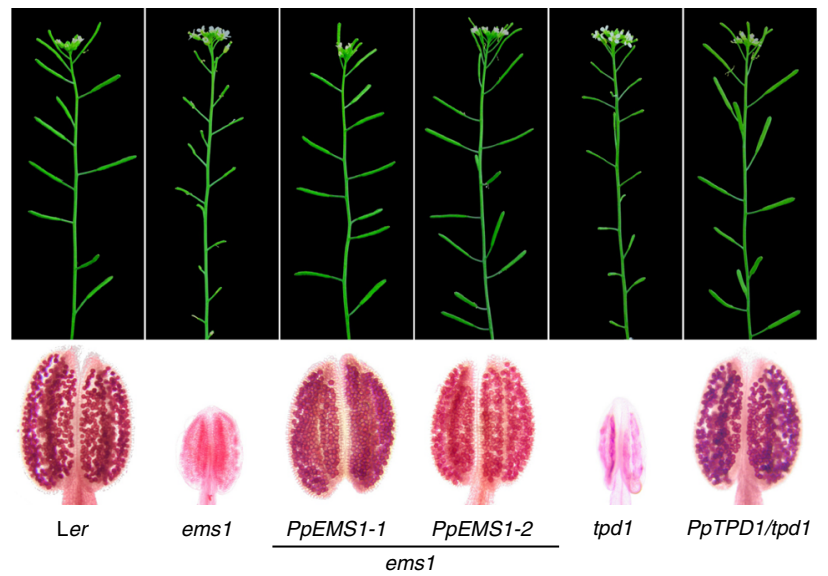

b

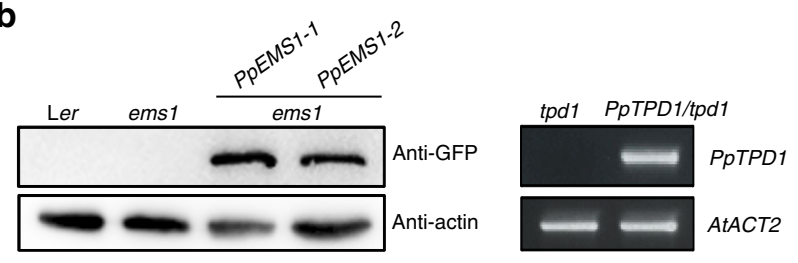

C

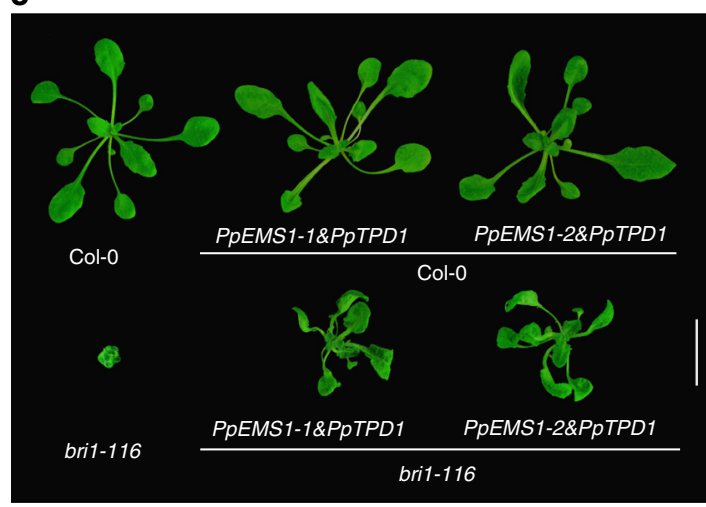

d

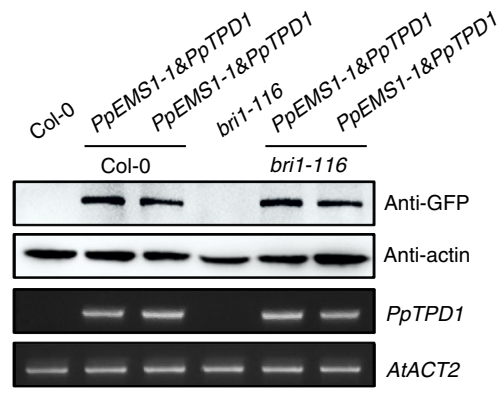

e

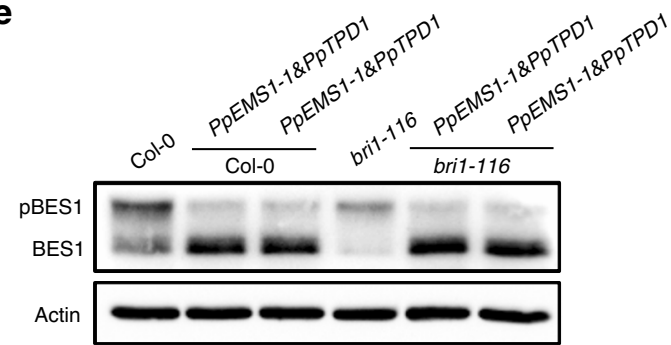

g

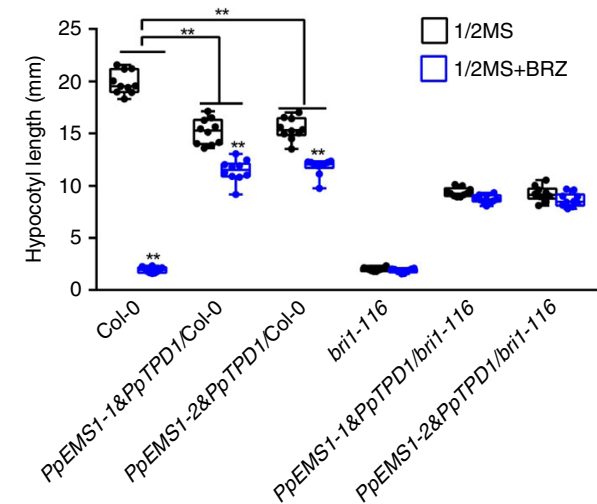

f

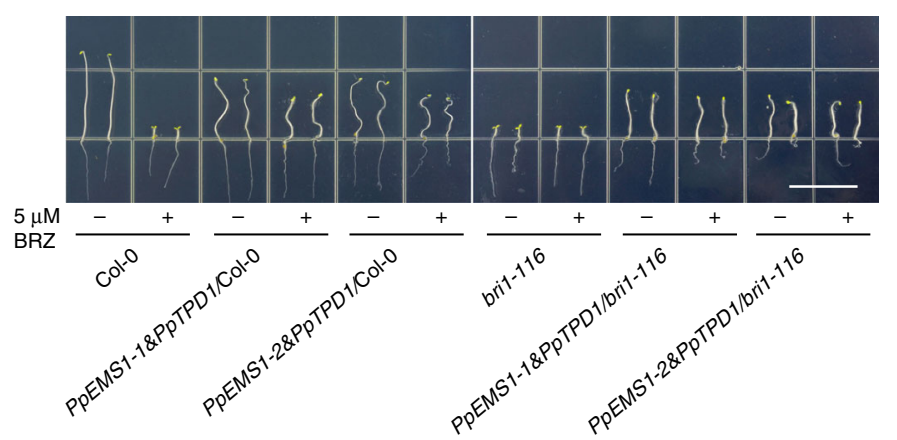

h

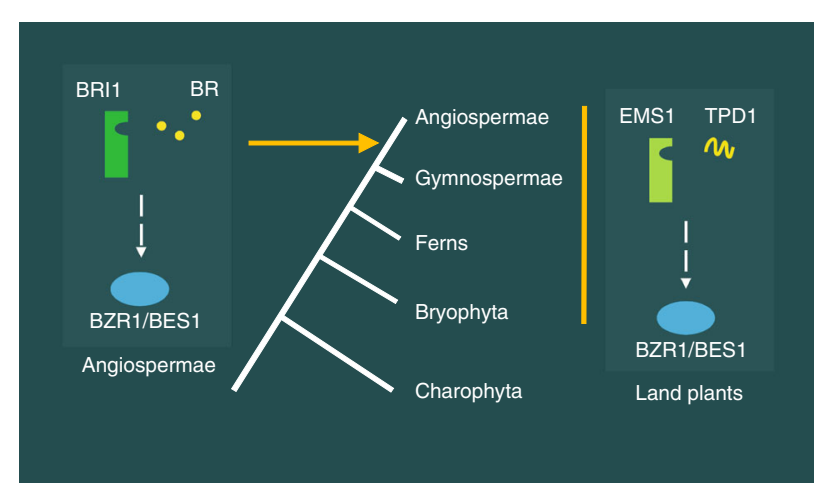

In the RLKs, the ECDs evolve faster than ICDs, so it is possible that ECDs have acquired the ability to perceive distinct signals, while the ICDs still target similar downstream molecules to control apparently diverse biological processes ${ }^{18,59}$. This allows us to swap the ECDs and ICDs to molecularly complement mutants between the RLKs with and without known signaling components. When complementation is phenotypically substantial, partly or completely in both directions, the tested RLKs are likely able to activate a common set of downstream molecular components when present in the reciprocal expression domains. In 
Fig. 4 PpEMS1 and PpTPD1 (from moss Physcomitrella patens) function as Arabidopsis EMS1 and TPD1 in Arabidopsis, respectively. a PpEMS1 and PpTPD1 completely restored the phenotypes of ems1 and tpd1, respectively. $\mathbf{b}$ Analyses of the expression levels of the transgenes in the inflorescences of the corresponding plants shown in a. Proteins with GFP tag were detected with anti-GFP antibody. Actin served as the loading control. PpTPD1 expression levels were detected by semi-quantitative RT-PCR, ACT2 served as an internal control. c Co-expressing PpEMS1 and PpTPD1 under the BR/1 promoter partially rescued bri1 phenotypes. Phenotypes of 4-week-old Col-0, PpEMS1-1 \& PpTPD1 and PpEMS1-2 \& PpTPD1 in Col-0 and bri1-116 mutants were shown, respectively. Scale bar, $2 \mathrm{~cm}$. d Analyses of the expression levels of the transgenes in the inflorescences of the corresponding plants shown in $\mathbf{c}$. Proteins with GFP tag were detected with anti-GFP antibody. Actin served as the loading control. PpTPD1 expression levels were detected by semi-quantitative RTPCR. ACT2 served as an internal control. e Co-expressing PpEMS1 and PPTPD1 under the BRI1 promoter in Col-0 and bri1-116 induced BES1 dephosphorylation. Phosphorylated BES1 (pBES1) and dephosphorylated BES1 were detected with BES1 antibodies in the extracts of 10-day-old seedlings of the indicated genotypes. Actin served as the loading control. $\mathbf{f}, \mathbf{g} 5$-day-old dark-grown seedlings in 1/2 MS medium with or without $5 \mu$ M BRZ. Scale bar, $1.5 \mathrm{~cm} . n=10$ seedlings. ${ }^{\star} P<0.0001$ (two-way ANOVA with Sidak's test). Co-expressing PpEMS1-1 \& PpTPD1 and PpEMS1-2 \& PpTPD1 in Col-0 or bri1-116 showed less sensitivity or insensitivity to BRZ, respectively. $\mathbf{h}$ A proposed model illustrating that EMS1 and BRI1 have evolved distinct extracellular domains to control different biological processes but can act via a common intracellular signaling pathway

our case, we made chimeric EMS1 and BRI1 receptors using their respective ECDs and ICDs, but theoretically this approach is not limited to the RLKs and could be applied to other diverged protein pairs.

It should be noted that our approach relies upon the ectopic expression of EMS or BRI1 in reciprocal expression domains. It is therefore conceivable that phenotypic rescue and correlated molecular events may not reflect the native function of the protein. For example, it is possible that EMS1-TPD1 mediated activation of BES1 occurs due to ectopic expression of EMS1 in the BRI1 expression domain. Likewise, dominant mutants of BES1/ BZR1 expressed in the tapetum might conceivably lead to effects that are not typical of native EMS1 signaling. Nevertheless, given that the complementation is reciprocal and that higher order BES1/BZR1 loss-of-function mutants demonstrate fertility defects $^{44}$, our analyses suggest that EMS1 shares common signaling components with BR signaling. In this cascade, the cell surface receptor EMS1 binds with small protein ligand TPD1 to activate downstream components ${ }^{7,12}$. We propose that this activates the BZR1 and BES1 transcription factors to regulate the expression of target genes to determinate the tapetum cell fate. Several lines of evidence support this model: (i) the functional domains of EMS1 and BRI1 can be exchangeable; (ii) EMS1TPD1 and BRI1-BR signaling can trigger similar molecular response when expressed in each other's expression domains; and (iii) dominant mutants bzr1-1D and bes1-D suppress both bri1 and ems 1 mutants. BRI1-BR signaling regulates almost all aspects of plant growth and development ${ }^{21,35,37,39}$, while EMS1-TPD1 signaling only controls tapetum determination $6,7,10,11$, therefore it may be surprising that such different biological processes could be controlled by similar molecular components. However, it may be the case that it is more economical for plants to use limited molecules to regulate a variety of plant development and cope with complex and variable internal and external environments.

A significant question is how did EMS1 and BRI1 evolve? One possibility is that EMS1 and BRI1 were duplicated and diverged from a common ancestor ${ }^{61-63}$. The ancestral duplicates have similar but tapetum-non-specific expression patterns and functions, which generates a potential adaptive conflict (optimizing one function compromising the other) requiring resolution ${ }^{64}$. In this case, a steroid binding site is thought to have gradually emerged in ancestral BR receptors ${ }^{56}$. When the ligand switches from a protein TPD1 of $20 \mathrm{kDa}$ to a chemical BR of only about $0.5 \mathrm{kDa}^{12,42}$, it could have allowed rapid evolution in the ECDs of ancestral BRI1, allowing BR activity to coevolve with BR-binding affinity in the ancestral BRI1. In complex tissues and organs, small ligands such as BRs may be easier to transport. Conversely, a large protein ligand such as TPD1 could be more easily confined to the space between the tapetal cell layer and the pollen mother cell than a small ligand that may diffuse to the neighboring cells resulting in functional imprecision, thus favoring the large ligand for communications between two cell layers ${ }^{12}$. Therefore, we propose that EMS1 perceives TPD1 in tapeta while BRI1 binds BRs elsewhere to control whole plant development in a complementary manner acting via a common downstream signaling pathway6,10,11,21. As a result, both EMS1 and BRI1 became essential in angiosperms $7,11,21,40,65$. Future study of the functional divergence of EMS1 and BRI1 could boost the understanding of the function and evolution of other RLKs.

Our study on EMS1 and BRI1 suggests that RLKs can perceive distinct ligands but trigger a common signaling pathway to control diverse biological and physiological processes. This suggests ligand-driven coevolution of RLKs in their ECDs could have resulted in diverse ECDs but conserved ICDs in RLKs.

\section{Methods}

Plant materials and growth conditions. The Arabidopsis thaliana Landsberg erecta (Ler) and Columbia (Col-0) ecotypes were used as wild type (WT) control in this study. ems 1 and tpd1 mutants are in the Ler background. bri1-301, bri1-116, det2, bin2-1D, serk1 (SALK 044330), serk2 (SALK 058020) and bzr1-1D alleles are in Col-0 background, and the mutant bes1-D is in Enkheim-2 (En-2). Seeds were germinated on either $1 \frac{1}{2}$ Murashige \& Skoog (MS) medium, then transferred to the soil or directly planted in the soil. Plants were grown under long daylight conditions (16-h light/8-h dark cycles). Seedlings for measurement of BR-dependent responses were germinated on $1 / 2$ MS medium with or without BL (Solarbio) or BRZ in the dark or in the light. Pictures of the plates were then taken for measurement of root length and hypocotyl length using ImageJ software.

Generation of constructs and transgenic plants. For genetic analyses, the BRII, $E M S 1$ and TPD1 promoters were individually introduced into the $p C H F 3$ (kanamycin selection) and PCAMBIA1300 (hygromycin B selection) plasmids with or without GFP to construct backbones listed in Supplementary Table 2. Primers for generating these backbones were included in Supplementary Table 1. The cDNA sequences of BRI1, EMS1, TPD1, PpTPD1 and the genomic sequences of PpEMS1s were inserted into the vectors' backbones to complement the mutants as indicated in the text or figures. Overlapping PCR was used to replace the ECD (extracellular domain) or ICD (intracellular domain) to generate $E C D^{B R I 1}: I C D^{R L K s}, E C D^{E M S 1}: I C D^{R L K s}$ and $E C D^{B R I 1}$ ICD PPEMS1s chimeric genes. The genomic sequences of bes $1-D$ and $b z r 1-1 D$ were cloned to activate BRI1 or EMS1 signaling pathway. They were then expressed in the Col-0, bri1-116, bri1-301, ems1, det2, or bin2-1D as indicated in the figures or text. All above constructs were transferred into plants via Agrobacterium (GV3101)-mediated transformation using the method described elsewhere ${ }^{66}$. The transformants were then screened on $1 / 2 \mathrm{MS}$ with $50 \mu \mathrm{g} / \mathrm{ml}$ kanamycin or $40 \mu \mathrm{g} / \mathrm{ml}$ hygromycin B. Double transgenic plants were produced by crossing, followed by a screen on $1 / 2$ MS with 50 $\mu \mathrm{g} / \mathrm{ml}$ kanamycin and $40 \mu \mathrm{g} / \mathrm{ml}$ hygromycin B. Primers and constructs used in this study were given in Supplementary Tables 1 and 2, respectively. Molecular genotyping was performed using the primers listed in Supplementary Table 1. At least 20 independent transgenic lines were obtained from each construct.

Protein extraction and immunoblot analysis. Total protein extracts were extracted from plant tissues with $2 \times$ SDS buffer (100 mM Tris, pH 6.8, 4\% [w/v] SDS, $20 \%[\mathrm{v} / \mathrm{v}]$ glycerol, $0.2 \%[\mathrm{w} / \mathrm{v}]$ bromophenol blue, $2 \%[\mathrm{v} / \mathrm{v}] \beta$-Mercaptoethanol), separated on SDS-PAGE gel, and then transferred to a Nitrocellulose membrane (Millipore). AntiGFP antibodies (1:1000 dilution, Transgen, HT801) were used to detect GFP fusion proteins and BES1 antibodies (1:3000 dilution, kindly provided by $\mathrm{J}$. Li, Lanzhou University, China) were used to detect the phosphorylation status of BES1. The equal loading was judged by Actin (1:1000 dilution, Abbkine A01050). 
Pollen staining and anther semi-thin sections. To observe viable pollen grains, anthers prior to dehiscence were fixed in Carnoy's fluid (alcohol: chloroform: acetic acid $=6: 3: 1$ ) for $2 \mathrm{~h}$. Then, the anthers were dissected and stained with Alexander's solution at $50{ }^{\circ} \mathrm{C}$ for $48 \mathrm{~h}^{67}$. Anthers were photographed under a compound microscope. For the anther structure study ${ }^{67}$, dissected floral buds and inflorescences were fixed in $4 \%(\mathrm{v} / \mathrm{v})$ glutaraldehyde in $0.1 \mathrm{M}$ PBS buffer $(\mathrm{pH} 6.8)$ and $0.02 \%(\mathrm{v} / \mathrm{v})$ Triton X-100 overnight at $4{ }^{\circ} \mathrm{C}$. Samples were washed 4 times for 15 min each in $0.1 \mathrm{M}$ PBS ( $\mathrm{pH} 6.8$ ) and then fixed in $1 \% \mathrm{OSO}_{4}$ for $4 \mathrm{~h}$. They were then dehydrated in a graded ethyl alcohol (20\% increments) and embedded in Spurr's resin. Semi-thin $(0.5 \mu \mathrm{m})$ sections were made by using a Leica EM UC7 Ultramicrotome (Leica Microsystems) and were stained with $0.25 \%$ of Toluidine Blue O. The images were photographed using an optronics digital camera. Tapetal cells and microsporocytes were determined under the microscope in the central sections of the anthers.

Microscopy analysis. Images of pollen staining and semi-thin sections were photographed under a Leica microscope equipped with a digital camera ${ }^{12}$. For confocal microscopy analysis, samples were observed under a Leica TCS SP5 laser scanning confocal microscope. They were then mounted in water and observed with a $\times 20$ lens or a $\times 40 / 1.1$ water immersion objective lens. A 488 -nm laser was used to excite GFP and chlorophyll. The emission was captured using PMTs set at 505-530 nm and 644-719 nm for GFP and chlorophyll, respectively.

qRT-PCR analysis. Total RNA was isolated from the leaves of 4-week-old plants or 6-week-old inflorescences using a HiPure Plant RNA Mini Kit (Magen, R4151-02) according to the protocol provided by the manufacturer. First-strand cDNA was synthesized from $1 \mu \mathrm{g}$ of total RNA using M-MLV First Strand cDNA Synthesis Kit (Omega, TQ2501-02). The qRT-PCR was performed using ChamQ ${ }^{\mathrm{TM}}$ SYBR qPCR Master Mix (Vazyme, Q311-00) to detect the transcript levels of genes. AtACTIN2 (AtACT2) was used as an internal control for qRT-PCR. The primers used are listed in Supplementary Table 1.

Statistical analysis. Statistical analysis was performed using one-way analysis of variance (ANOVA), two-way ANOVA and Tukey's test or Sidak's test, as implemented in GraphPad Prism 6.0 (GraphPad Software, http://www.graphpad.com).

Sequence data. Sequence data for this article can be found in the TAIR (https:// www.arabidopsis.org/), Phytozome 12 database (https://phytozome.jgi.doe.gov/pz/ portal.html\#), congenie.org (http://congenie.org/) and FernBase (https://www. fernbase.org/) under the following accession numbers (all genes are from Arabidopsis thaliana except otherwise indicated):

BRI1, AT4G39400; EMS1, AT5G07280; TPD1, AT4G24972; BRL1, AT1G55610; BRL3, AT3G13380; BRL2, AT1G14000; PSKR1, AT2G02220; GSO1, AT4G20140; CLV1, AT1G75820; EFR, AT5G20480; BAM1, AT5G65700; BAK1, AT4G33430; CTR1, AT5G03730; BIN2, AT4G18710; BES1, AT1G19350; BZR1, AT1G75080; ACT2, AT3G18780; CPD, AT5G05690; DWF4, AT3G50660; BAS1, AT2G26710; SERK1, AT1G71830; SERK2, AT1G34210; OsMSP1, LOC_Os01g68870.1; AmEMS1, evm_27.model.AmTr_v1.0_scaffold00009.24; PaEMS1, MA_1913g0010; AfEMS1, Azfi_s0017.g014644; ScEMS1, Sacu_v1.1_s0054.g014268; SmEMS1, Sm99902; PpEMS1-1, Pp3c1_41620V3.1; PpEMS1-2, Pp3c14_16840V3.1; PpEMS1-3, Pp3c22_12040V3.1; PpEMS1-4, Pp3c19_18410V3.2; PpEMS1-5, Pp3c17_21540V3.3; PpEMS1-6, Pp3c1_16110V3.4; MpEMS1, Mapoly0011s0213.1; OsTPL1A, LOC Os12g28750.1; AmTPD1, evm_27.model.AmTr_v1.0_scaffold00047.41; PaTPD1, MA_10427288g0010; AfTPD1, Azfi_s0003.g008001; ScTPD1, Sacu_v1.1_ s0032.g010785; SmTPD1, Sm113463; PpTPD1, Pp3c22_22420V3.1; MpTPD1, Mapoly0020s0056.1. AT: Arabidopsis thaliana; Os: Oryza sativa; Am, Amborella trichopoda; Pa, Picea abies; Af, Azolla filiculoides; Sc, Salvinia cucullata; Pp: Physcomitrella patens; Sm: Selaginella moellendorffi; Mp: Marchantia polymorpha.

Reporting summary. Further information on research design is available in the Nature Research Reporting Summary linked to this article.

\section{Data availability}

The authors declare that all data supporting the findings of this study are available within the manuscript and its supplementary files, or are available from the corresponding authors upon request. The source data underlying Figs. 1-4 and Supplementary Figs. 219 are provided as a Source Data file.

Received: 10 January 2019 Accepted: 22 August 2019

Published online: 13 September 2019

\section{References}

1. Zhang, D., Luo, X. \& Zhu, L. Cytological analysis and genetic control of rice anther development. J. Genet. Genomics 38, 379-390 (2011).
2. Ma, H. Molecular genetic analyses of microsporogenesis and microgametogenesis in flowering plants. Annu. Rev. Plant Biol. 56, 393-434 (2005).

3. Goldberg, R. B., Beals, T. P. \& Sanders, P. M. Anther development: basic principles and practical applications. Plant Cell 5, 1217-1229 (1993).

4. Cai, W. \& Zhang, D. The role of receptor-like kinases in regulating plant male reproduction. Plant Reprod. 31, 77-87 (2018)

5. Feng, X. \& Dickinson, H. G. Tapetal cell fate, lineage and proliferation in the Arabidopsis anther. Development 137, 2409-2416 (2010).

6. Yang, S. L. et al. Tapetum determinant 1 is required for cell specialization in the Arabidopsis anther. Plant Cell 15, 2792-2804 (2003).

7. Jia, G., Liu, X., Owen, H. A. \& Zhao, D. Signaling of cell fate determination by the TPD1 small protein and EMS1 receptor kinase. Proc. Natl Acad. Sci. USA 105, 2220-2225 (2008).

8. Chang, F., Wang, Y., Wang, S. \& Ma, H. Molecular control of microsporogenesis in Arabidopsis. Curr. Opin. Plant Biol. 14, 66-73 (2011).

9. $\mathrm{Xu}$, J. et al. ABORTED MICROSPORES acts as a master regulator of pollen wall formation in Arabidopsis. Plant Cell 26, 1544-1556 (2014).

10. Zhao, D. Z., Wang, G. F., Speal, B. \& Ma, H. The excess microsporocytes 1 gene encodes a putative leucine-rich repeat receptor protein kinase that controls somatic and reproductive cell fates in the Arabidopsis anther. Genes Dev. 16, 2021-2031 (2002).

11. Canales, C., Bhatt, A. M., Scott, R. \& Dickinson, H. EXS, a putative LRR receptor kinase, regulates male germline cell number and tapetal identity and promotes seed development in Arabidopsis. Curr. Biol. 12, 1718-1727 (2002).

12. Huang, J. et al. Control of anther cell differentiation by the small protein ligand TPD1 and its receptor EMS1 in Arabidopsis. PLoS Genet. 12, e1006147 (2016).

13. Li, Z. et al. Two SERK receptor-like kinases interact with EMS1 to control anther cell fate determination. Plant Physiol. 173, 326-337 (2017).

14. Vidalain, P. O., Boxem, M., Ge, H., Li, S. \& Vidal, M. Increasing specificity in high-throughput yeast two-hybrid experiments. Methods 32, 363-370 (2004).

15. Gavin, A. C. et al. Functional organization of the yeast proteome by systematic analysis of protein complexes. Nature 415, 141-147 (2002).

16. Miernyk, J. A. \& Thelen, J. J. Biochemical approaches for discovering protein protein interactions. Plant J. 53, 597-609 (2008).

17. Huang, J. et al. Carbonic anhydrases function in anther cell differentiation downstream of the receptor-like kinase EMS1. Plant Cell 29, 1335-1356 (2017).

18. Shiu, S. H. et al. Comparative analysis of the receptor-like kinase family in Arabidopsis and rice. Plant Cell 16, 1220-1234 (2004).

19. Shiu, S. H. \& Bleecker, A. B. Receptor-like kinases from Arabidopsis form a monophyletic gene family related to animal receptor kinases. Proc. Natl Acad. Sci. USA 98, 10763-10768 (2001).

20. Sauter, M. Phytosulfokine peptide signalling. J. Exp. Bot. 66, 5161-5169 (2015).

21. Li, J. \& Chory, J. A putative leucine-rich repeat receptor kinase involved in brassinosteroid signal transduction. Cell 90, 929-938 (1997).

22. He, Z. et al. Perception of brassinosteroids by the extracellular domain of the receptor kinase BRI1. Science 288, 2360-2363 (2000).

23. Hohmann, U. et al. Mechanistic basis for the activation of plant membrane receptor kinases by SERK-family coreceptors. Proc. Natl Acad. Sci. USA 115, 3488-3493 (2018).

24. Kinoshita, T. et al. Binding of brassinosteroids to the extracellular domain of plant receptor kinase BRI1. Nature 433, 167-171 (2005).

25. Wang, Z. Y., Seto, H., Fujioka, S., Yoshida, S. \& Chory, J. BRI1 is a critical component of a plasma-membrane receptor for plant steroids. Nature 410, 380-383 (2001).

26. Clouse, S. D. Brassinosteroids. Arabidopsis Book 9, e0151 (2011).

27. $\mathrm{Li}$, J. et al. BAK1, an Arabidopsis LRR receptor-like protein kinase, interacts with BRI1 and modulates brassinosteroid signaling. Cell 110, 213-222 (2002)

28. Wu, G. et al. Methylation of a phosphatase specifies dephosphorylation and degradation of activated brassinosteroid receptors. Sci. Signal. 4, ra29 (2011).

29. Nam, K. H. \& Li, J. BRI1/BAK1, a receptor kinase pair mediating brassinosteroid signaling. Cell 110, 203-212 (2002).

30. Wang, X. \& Chory, J. Brassinosteroids regulate dissociation of BKI1, a negative regulator of BRI1 signaling, from the plasma membrane. Science 313, 1118-1122 (2006)

31. Tang, W. et al. BSKs mediate signal transduction from the receptor kinase BRI1 in Arabidopsis. Science 321, 557-560 (2008).

32. Li, J., Nam, K. H., Vafeados, D. \& Chory, J. BIN2, a new brassinosteroidinsensitive locus in Arabidopsis. Plant Physiol. 127, 14-22 (2001).

33. He, J. X., Gendron, J. M., Yang, Y., Li, J. \& Wang, Z. Y. The GSK3-like kinase BIN2 phosphorylates and destabilizes BZR1, a positive regulator of the brassinosteroid signaling pathway in Arabidopsis. Proc. Natl Acad. Sci. USA 99, 10185-10190 (2002).

34. Mora-Garcia, S. et al. Nuclear protein phosphatases with Kelch-repeat domains modulate the response to brassinosteroids in Arabidopsis. Genes Dev. 18, 448-460 (2004). 
35. Yin, Y. et al. BES1 accumulates in the nucleus in response to brassinosteroids to regulate gene expression and promote stem elongation. Cell 109, 181-191 (2002).

36. Wang, Z. Y. et al. Nuclear-localized BZR1 mediates brassinosteroid-induced growth and feedback suppression of brassinosteroid biosynthesis. Dev. Cell 2, 505-513 (2002).

37. He, J. X. et al. BZR1 is a transcriptional repressor with dual roles in brassinosteroid homeostasis and growth responses. Science 307, 1634-1638 (2005).

38. Yin, Y. et al. A new class of transcription factors mediates brassinosteroidregulated gene expression in Arabidopsis. Cell 120, 249-259 (2005).

39. Ye, Q. et al. Brassinosteroids control male fertility by regulating the expression of key genes involved in Arabidopsis anther and pollen development. Proc. Natl Acad. Sci. USA 107, 6100-6105 (2010).

40. Szekeres, M. et al. Brassinosteroids rescue the deficiency of CYP90, a cytochrome P450, controlling cell elongation and de-etiolation in Arabidopsis. Cell 85, 171-182 (1996).

41. Asami, T. et al. Characterization of brassinazole, a triazole-type brassinosteroid biosynthesis inhibitor. Plant Physiol. 123, 93-100 (2000).

42. Clouse, S. D. A history of brassinosteroid research from 1970 through 2005: thirty-five years of phytochemistry, physiology, genes, and mutants. J. Plant Growth Regul. 34, 828-844 (2015).

43. Grove, M. D. et al. Brassinolide, a plant growth-promoting steroid isolated from Brassica napus pollen. Nature 281, 216-217 (1979).

44. Chen, L. G., et al. BZR1 family transcription factors function redundantly and indispensably in BR signaling but exhibit BRI1-independent function in regulating anther development in Arabidopsis. Mol. Plant, https://doi.org/ 10.1016/j.molp.2019.06.006 (2019).

45. Rensing, S. A. et al. The Physcomitrella genome reveals evolutionary insights into the conquest of land by plants. Science 319, 64-69 (2008).

46. Bowman, J. L. et al. Insights into land plant evolution garnered from the marchantia polymorpha genome. Cell 171, 287-304.e215 (2017).

47. Li, F. W. et al. Fern genomes elucidate land plant evolution and cyanobacterial symbioses. Nat. Plants 4, 460-472 (2018).

48. Nystedt, B. et al. The Norway spruce genome sequence and conifer genome evolution. Nature 497, 579-584 (2013).

49. Guan, R. et al. Draft genome of the living fossil Ginkgo biloba. Gigascience 5, 49 (2016).

50. Nordberg, H. et al. The genome portal of the Department of Energy Joint Genome Institute: 2014 updates. Nucleic Acids Res. 42, D26-D31 (2014).

51. Merchant, S. S. et al. The Chlamydomonas genome reveals the evolution of key animal and plant functions. Science 318, 245-250 (2007).

52. Cano-Delgado, A. et al. BRL1 and BRL3 are novel brassinosteroid receptors that function in vascular differentiation in Arabidopsis. Development 131 5341-5351 (2004).

53. Zhou, A., Wang, H., Walker, J. C. \& Li, J. BRL1, a leucine-rich repeat receptorlike protein kinase, is functionally redundant with BRI1 in regulating Arabidopsis brassinosteroid signaling. Plant J. 40, 399-409 (2004).

54. Clay, N. K. \& Nelson, T. VH1, a provascular cell-specific receptor kinase that influences leaf cell patterns in Arabidopsis. Plant Cell 14, 2707-2722 (2002).

55. Ju, C. et al. Conservation of ethylene as a plant hormone over 450 million years of evolution. Nat. Plants 1, 14004 (2015).

56. Wang, H. \& Mao, H. On the origin and evolution of plant brassinosteroid receptor kinases. J. Mol. Evol. 78, 118-129 (2014).

57. Banks, J. A. et al. The Selaginella genome identifies genetic changes associated with the evolution of vascular plants. Science 332, 960-963 (2011).

58. Shiu, S. H. \& Bleecker, A. B. Expansion of the receptor-like kinase/Pelle gene family and receptor-like proteins in Arabidopsis. Plant Physiol. 132, 530-543 (2003).

59. Shiu, S. H. \& Bleecker, A. B. Plant receptor-like kinase gene family: diversity, function, and signaling. Sci. STKE 2001, re22 (2001).

60. Jiang, J., Zhang, C. \& Wang, X. Ligand perception, activation, and early signaling of plant steroid receptor brassinosteroid insensitive 1. J. Integr. Plant Biol. 55, 1198-1211 (2013).
61. Ohno, S. Evolution by Gene Duplication. (Allen \& Unwin, Springer-Verlag, 1970).

62. Force, A. et al. Preservation of duplicate genes by complementary, degenerative mutations. Genetics 151, 1531-1545 (1999).

63. Hughes, A. L. The evolution of functionally novel proteins after gene duplication. Proc. Biol. Sci. 256, 119-124 (1994).

64. Hittinger, C. T. \& Carroll, S. B. Gene duplication and the adaptive evolution of a classic genetic switch. Nature 449, 677-681 (2007).

65. Rancati, G., Moffat, J., Typas, A. \& Pavelka, N. Emerging and evolving concepts in gene essentiality. Nat. Rev. Genet. 19, 34-49 (2018).

66. Zhang, X., Henriques, R., Lin, S. S., Niu, Q. W. \& Chua, N. H. Agrobacteriummediated transformation of Arabidopsis thaliana using the floral dip method. Nat. Protoc. 1, 641-646 (2006).

67. Alexander, M. P. Differential staining of aborted and nonaborted pollen. Stain Technol. 44, 117-122 (1969).

\section{Acknowledgements}

We thank H. Ma for ems1, J. Li (U. Michigan) for bin2-1D, Z.Y. Wang for bzr1-1D, Y. Yin for bes1-D and J. Li (Lanzhou University) for serk1serk2 (serk1/2) and BES1 antibodies. Financial support to G.W. from Shaanxi Normal University, Chinese Ministry of Education (313034) and (20130202110007); Fundamental Research Funds for Central Universities to G.W. (GK201101005), B.Z. (2017CB2004) and Q.B. (2017TS032); Grants from the Chinese National Foundation of Science to G.W. (31270324 and 31741014), and postdoctoral fellowships from Chinese National Foundation of Science to H.R (2012M521740) and G.S.L. (2012M521739).

\section{Author contributions}

G.W. and X.S. conceived and guided the study. B.Z., Q.B., X.S., and G.W. designed the experiments. Q.B., B.Z., L.W., H.L., and Y.L. performed the experiments. B.Z., Q.B., L.W., H.L., Y.L., G.L., W.X., H.R., X.S., and G.W. analyzed the data and interpreted the results. G.W. wrote the manuscript, and the other authors read and edited the manuscript.

\section{Additional information}

Supplementary Information accompanies this paper at https://doi.org/10.1038/s41467019-12112-w.

Competing interests: The authors declare no competing interests.

Reprints and permission information is available online at http://npg.nature.com/ reprintsandpermissions/

Peer review information: Nature Communications thanks Eugenia Russinova and the other, anonymous, reviewer(s) for their contribution to the peer review of this work. Peer reviewer reports are available.

Publisher's note: Springer Nature remains neutral with regard to jurisdictional claims in published maps and institutional affiliations.

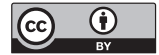

Open Access This article is licensed under a Creative Commons Attribution 4.0 International License, which permits use, sharing, adaptation, distribution and reproduction in any medium or format, as long as you give appropriate credit to the original author(s) and the source, provide a link to the Creative Commons license, and indicate if changes were made. The images or other third party material in this article are included in the article's Creative Commons license, unless indicated otherwise in a credit line to the material. If material is not included in the article's Creative Commons license and your intended use is not permitted by statutory regulation or exceeds the permitted use, you will need to obtain permission directly from the copyright holder. To view a copy of this license, visit http://creativecommons.org/ licenses/by/4.0/.

(C) The Author(s) 2019 